\title{
Voluminous Laryngeal Schwannoma Treated with Endoscopic Laser Approach
}

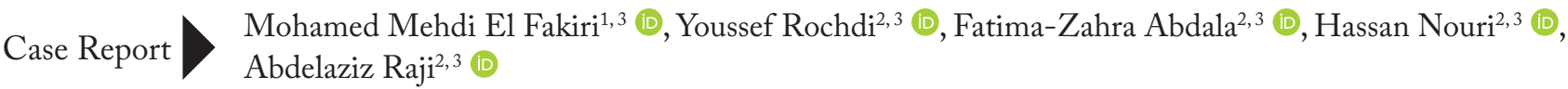 \\ ${ }^{1}$ Department of Anatomy, Ibn Zohr University, School of Medicine and Pharmacy, Agadir, Morocco \\ ${ }^{2}$ Department of Head and Neck Surgery, Cadi Ayyad University, School of Medicine and Pharmacy, Marrakesh, Morocco \\ ${ }^{3}$ Department of Otorhinolaryngology, Mohammed VI University Hospital Center, Marrakesh, Morocco
}

Abstract

ORCID IDs of the authors: M.M.E.F. 0000-0003-2931-8453; Y.R.:0000-0001-7410-9672; F.Z.A.:0000-0001-5183-7263; H.R.: 0000-0002-4410-9476; A.R.:0000-0002-9783-724X.

Cite this article as: El Fakiri MM, Rochdi Y, Abdala FZ, Nouri H, Raji A. Voluminous Laryngeal Schwannoma Treated with

Endoscopic Laser Approach. Turk Arch Otorhinolaryngol 2020; 58(1): 52-5.

Corresponding Author:

Mohamed Mehdi El Fakiri, elfakirimehdi@yahoo.fr

Received Date: 25.06.2019

Accepted Date: 06.12.2019

Content of this journal is licensed under a Creative Commons Attribution 4.0 International License. Available online at www.turkarchotolaryngol.net
Laryngeal schwannomas are rare benign encapsulated neurogenic tumors that represent less than $1.5 \%$ of all benign laryngeal tumors. We report a case of voluminous laryngeal schwannoma that was incidentally found during endotracheal intubation for thyroidectomy in a 43-year-old woman with clinical findings, features of radiologic and histopathologic examinations. The tumor was removed by $\mathrm{CO}_{2}$ laser during microlaryngoscopy. In this case report, we present a challenging approach that can be used in diagnosis and treatment of laryngeal schwannomas. Complete removal of the tumor should be considered as the initial approach to minimize morbidity.

Keywords: Schwannoma, nerve sheath tumors, larynx, minimally invasive surgical procedures

\section{Introduction}

Schwannomas (neurilemmomas) are benign tumors arising from the Schwann cells in the peripheral nerve. These neurogenic tumors are rare, accounting for less than $1.5 \%$ of all benign laryngeal tumors, and usually present as insidious, slow-growing and submucosal masses (1).

This study presents a challenging approach that can be used in diagnosis and treatment. We report a case of bulky laryngeal schwannoma and its treatment with laser microlaryngoscopy.

\section{Case Presentation}

A 43-year-old female patient with a medical history of breast cancer and goiter had received treatment for her breast cancer for one year and six months later underwent thyroidectomy. A supraglottic mass was incidentally found during endotracheal intubation for thyroidectomy. Her Ear Nose and Throat (ENT) specialist had recommended investigation and treatment; however, the patient did not proceed with the treatment. Four months later the patient presented with progressive snoring and uncommon sensation in the throat.
Fiber-optic laryngoscopy revealed a bulky submucosal mass on the left aryepiglottic fold, extending to the left arytenoid and slightly obstructing the laryngeal respiratory space. Mobility of the vocal cords was normal (Figure 1). No cervical lymph nodes were palpable.

Neck computed tomography (CT) scan showed a mass measuring $18 \times 21 \times 23 \mathrm{~mm}$. It extended from the left aryepiglottic fold to the left arytenoid cartilage and the medial part of the left piriformis recess. The mass was hypodense but enhanced heterogeneously in contrast images (Figure 2). Magnetic Resonance Imaging (MRI) showed a well-delimited mass centered in the left aryepiglottic fold, hypointense signal in T1, hyperintense signal in T2 and hyperintense signal in T1-weighted images with gadolinium (Figure 3, 4).

Differential diagnoses were metastasis of breast cancer, Hodgkin's lymphoma, hemangioma adenoma and lipoma.

Laryngoscopy was performed under general anaesthesia. It showed a well-circumscribed, 
submucosal mass. The mass was sessile. Glottic and subglottic areas were normal. Deep biopsies of the mass suggested a diagnosis of schwannoma. Immunohistochemistry confirmed the diagnosis by strong immunoreactivity of tumor cells for S100 protein.

Primary tracheotomy was performed to avoid the potential risks of endotracheal intubation as well as a possible airway obstruction induced by post-operative oedema. The tumor was completely removed using $\mathrm{CO}_{2}$ laser microlaryngoscopy, thus the integrity of the capsule was maintained. The adjacent cartilage was preserved. The mucosa was subsequently closed with resorbable sutures, Histopathologic examination of the mass confirmed the diagnosis of schwannoma (Figure 5). Postoperative course

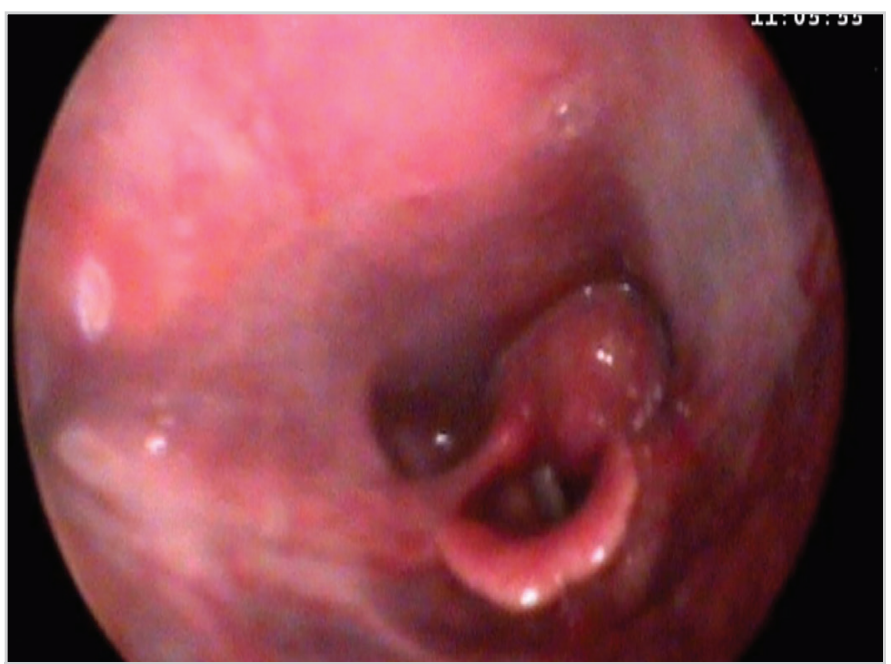

Figure 1. Fiberoptic laryngoscopy revealing a submucosal mass of the left aryepiglottic fold



Figure 2. Axial view of CT scan of the supraglottis showing heterogeneous image obliterating the left piriform recess was uneventful. Oral feeding was restored and the nasogastric tube was removed on the third day. Decannulation was done five days after surgery. Laryngeal morphology and motility have been preserved (Figure 6). Follow up was made for nine months without any recurrence.

\section{Discussion}

Laryngeal schwannomas are rare benign encapsulated neurogenic tumors that account for less than $1.5 \%$ of all benign laryngeal tumors (1). The most common anatomical site of laryngeal

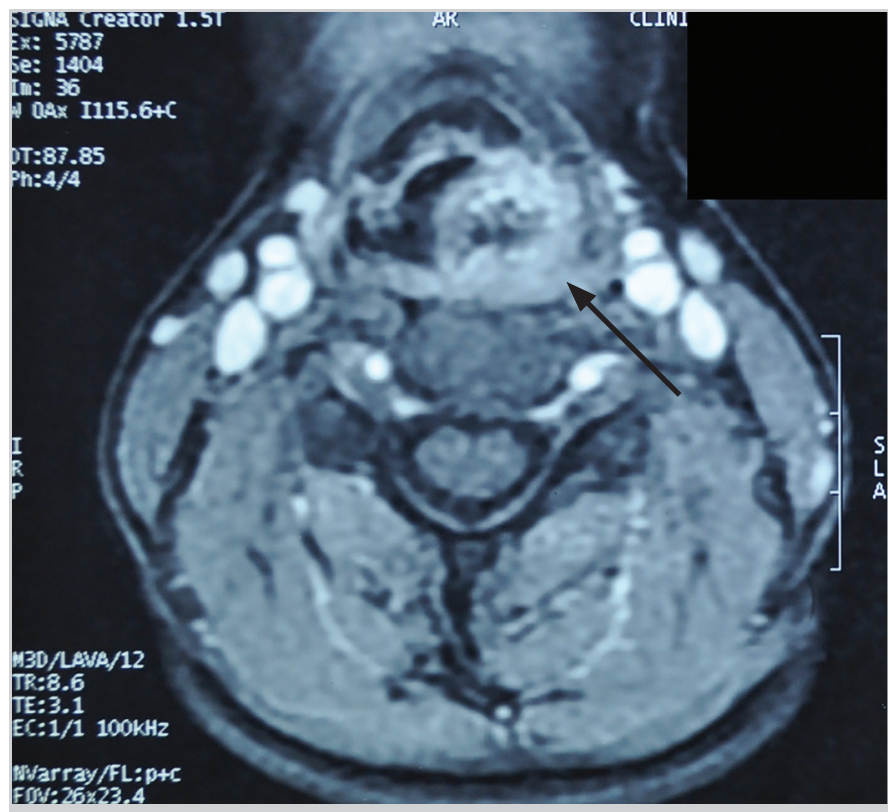

Figure 3. Axial view of cervical MRI showing a mass occupying glottic space, originating from the left aryepiglottic fold with hyperintense in T1-weighted images with gadolinium (black arrow)



Figure 4. Axial view of cervical MRI showing the mass with hyperintense in T2-weighted images (black arrow) 


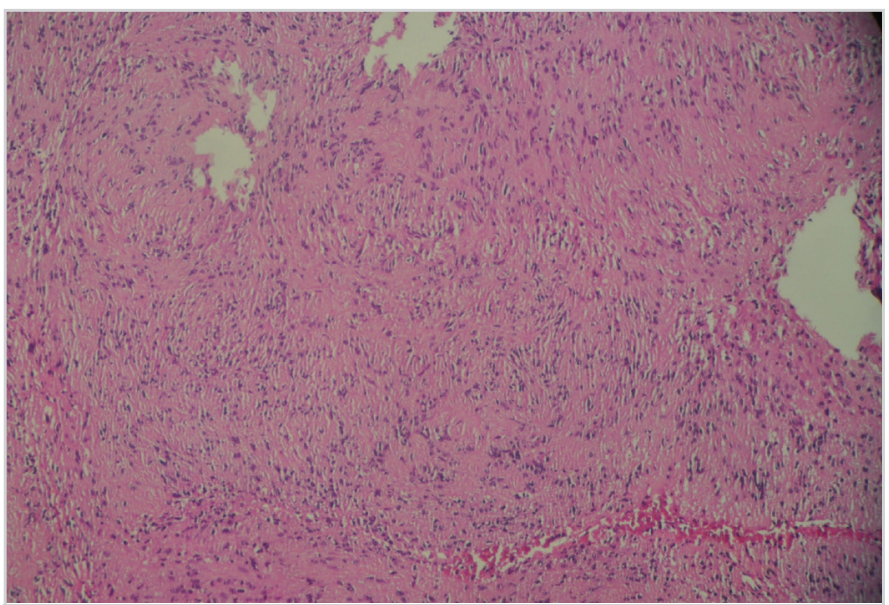

Figure 5. Histopathological image showing the characteristic of schwannoma with palisade-like structures and a cellular depletion $(\mathrm{x} 40)$



Figure 6. Fiberoptic laryngoscopy 15 days after surgery

schwannomas is the aryepiglottic fold (80\%), followed by the arytenoids, the ventricular folds and the vocal cords (20\%) (2). Laryngeal schwannomas are most commonly found in females in the $4^{\text {th }}$ to the $5^{\text {th }}$ decade of life (3).

They are usually solitary, generally occur with non-specific symptoms and are determined by their size and location. They can cause globus sensation, dysphagia, dysphonia. Bulky or pedunculated tumors are dangerous since they can lead to acute respiratory distress (4).

Classically, submucosal tumors appear on laryngoscopy as smooth lesions. Mass lesions may obstruct the view of the laryngeal inlet and result in reduced mobility of the vocal cord due to "pseudo-fixation" of the cricoarytenoid joint $(5,6)$.

Imaging is helpful for determining the location and the extension of the lesion but cannot differentiate it from other benign laryngeal tumors. Typically, MRI shows a well-demarcated lesion with round or oval shape, without infiltrative or destructive signs and heterogeneously enhanced on gadolinium-based contrast $(1,2)$.

Histologically, the tumor fulfilled Enzinger and Weiss's diagnosis criteria of schwannoma: the presence of a capsule, the presence of Antoni A and/or Antoni B areas, and S-100 protein positivity (1). Some authors prefer excisional biopsy since it allows for simultaneous diagnosis and definitive treatment as opposed to incisional biopsy which induces a higher risk of vocal cord paralysis or recurrence (7).

We have performed incisional biopsy first, to eliminate metastatic disease or a lymphoma. The appropriate curative treatment to prevent recurrence and to avoid damage to the healthy structures of the larynx is to proceed to a wide and complete surgical resection. There are various surgical methods. The best approach is to consider the preference of the patient, as well as the size and the location of the tumor. The pedunculated tumor can be easily excised by endoscopic approach. A trans-oral microlaryngoscopic excision with or without laser is the best choice for small or superficial tumors. However, indications for the endoscopic trans-oral approach have been recently extended with the advancement of surgical techniques that allow direct visualization and manipulation of the lesion with a reduced risk of damaging the surrounding structures (4). $\mathrm{CO}_{2}$ lasers are more precise than scalpels and the high temperature generated by the laser aids in cleaning the edges of the body tissue, and in reducing the risks of infection and recurrence. This technique is useful as it enables reducing surgery time as well as speeding up the healing time (8). External approaches include the median thyrotomy, the lateral pharyngotomy and the laryngofissure techniques. They are used in larger tumors or in cases which complete extirpation of the lesion is impossible by endoscopy (7-9). Sometimes, a tracheotomy may be required to secure the airway (5).

\section{Conclusion}

Laryngeal schwannoma is uncommon. Imaging helps to determine the localization and the size of the tumor and plan the surgical approach. Endoscopic surgery should be the initial approach to completely remove the tumor to minimize morbidity for the appropriate tumor size and location. Otherwise, enucleation can be done by external approach.

Informed Consent: Written informed consent was obtained from the patient who participated in this study.

Peer-review: Externally peer-reviewed.

Author Contributions: Concept - A.R., M.M.E.F.; Design A.R., H.N., M.M.E.F.; Supervision - A.R.; Resource - A.R., Y.R., M.M.E.F.; Materials - M.M.E.F., F.Z.A.; Data Collection and/or Processing - Y.R., M.M.E.F.; Literature Search - M.M.E.F., F.Z.A.; Writing - H.N., Y.R., M.M.E.F.; Critical Reviews - A.R., H.N., Y.R.

Conflict of Interest: The authors have no conflicts of interest to declare.

Financial Disclosure: The authors declared that this study has received no financial support.

\section{References}

1. Tulli M, Bondi S, Bussi M. Voluminous laryngeal schwannoma excision with a mini-invasive external approach: A case report. Acta Otorhinolaryngol Ital 2017; 37: 242-4.

2. Romak JJ, Neel HB 3rd, Ekbom DC. Laryngeal schwannoma: A case presentation and review of the Mayo Clinic experience. J Voice 2017; 31: 129.e15-129.e18. [Crossref] 
3. Bradley PJ. Benign neoplasms and other tumours of the larynx. Anniko M, Bernal-Sprekelsen M, Bonkowsky V. Bradley PJ, Lurato S, editors. Otorhinolaryngology, Head and Neck Surgery. Berlin, Heidelberg: Springer; 2010.p.487-97. [Crossref]

4. Mannarini L, Morbini P, Bertino G, Gatti O, Benazzo M. Acute respiratory distress in patient with laryngeal schwannoma. Case Rep Med 2012; 2012: 616913. [Crossref]

5. Markou K, Dova S, Poulios C, Karkos P. Laryngeal schwannoma as an acute airway presentation. BMJ Case Rep 2016; 2016: bcr2016214682. [Crossref]
6. Rosen FS, Pou AM, Quinn FB Jr. Obstructive supraglottic schwannoma: A case report and review of the literature. Laryngoscope 2002; 112: 997-1002. [Crossref]

7. Tulli M, Bondi S, Smart CE, Giordano L, Trimarchi M, Galli A, et al. Diagnosis and treatment of laryngeal schwannoma: A systematic review. Otolaryngol Head Neck Surg 2018; 158: 222-31. [Crossref]

8. Kou W, Zhang C, Wei P. Laryngeal schwannoma treated with a $\mathrm{CO}_{2}$ laser: A case report. Oncol Lett 2015; 9: 1467-9. [Crossref]

9. Tse A, Anwar B. Laryngeal schwannoma: Excision via a laryngofissure approach.J Surg Case Rep 2015; 2015: rjv059 [Crossref] 